\title{
Features of electrocardiogram in patients with stenosis of the proximal right coronary artery
}

\author{
Moo Seong Koh, Jae Hoon Lee, Jin Woo Jeong, and Jun Young Chung
}

Department of Emergency Medicine, Dong-A University College of

Medicine, Busan, Korea

\author{
Received: April 26, 2015 \\ Revised : June 26, 2015 \\ Accepted: September 14, 2015

\section{Correspondence to} \\ Jae Hoon Lee, M.D. \\ Department of Emergency \\ Medicine, Dong-A University \\ College of Medicine, 26 \\ Daesingongwon-ro, Seo-gu, \\ Busan 49201, Korea \\ Tel: +82-51-240-5590 \\ Fax: +82-51-240-5309 \\ E-mail: leetoloc@dau.ac.kr
}

Background/Aims: Prediction of lesions of the proximal right coronary artery (pRCA) through electrocardiogram (ECG) is very important because pRCA occlusion has many complications and a high mortality rate, which has frequently been related with right ventricular infarction. The purpose of this study was to devise a screening tool that takes into account multiple leads from a 12-lead ECG to predict the pRCA lesion.

Methods: A hundred and fifty-eight patients who were diagnosed as acute coronary syndrome and had a pure lesion of RCA or left circumflex artery (LCX) by ECGs and angiographic findings were enrolled retrospectively. Forty-eight patients with a pure pRCA occlusion were compared to a control group of 110 patients who were diagnosed as having either a pure mid to distal RCA lesion (57 patients) or a pure LCX lesion (53 patients).

Results: ECGs of patients in the pRCA group showed more prominent ST depression in lead I $(p=0.001)$ and ST elevation in $\mathrm{V}_{1}(p=0.002)$ than in the control group. The combination of ST depression $(\leq 0 \mathrm{~mm})$ in I and ST elevation $(>0.5$ $\mathrm{mm}$ ) in V1 was the best diagnostic tool (area under the curve, o.84).

Conclusions: ST changes in leads V1 and I allow more accurate prediction of pRCA occlusion than other criteria, such as the difference between ST elevation of leads II and III or vector direction and amplitude. These variables could help to screen for right ventricular infarction before performing reverse ECG and predicting prognosis.

Keywords: Coronary artery disease; Myocardial infarction; Electrocardiography; Coronary angiography

\section{INTRODUCTION}

The electrocardiogram (ECG) is the primary diagnostic tool used to evaluate and guide the treatment of patients with chest pain and suspected acute myocardial infarction (MI) in emergency departments (EDs). Physicians make decision about performing immediate angiography, using either early invasive strategy or a conservative strategy based upon clinical assessments, including a history of recurrent angina symptoms, elevated cardiac biomarkers, previous history, dynamic ECG, echocardiographic findings of heart dysfunction, and high risk scores [1]. A new ST-segment depression, which is considered a risk factor, and ST changes in specific leads may indicate serious problems, such as right ventricular (RV) injury.

Proximal right coronary artery (pRCA) occlusion usually leads to RV involvement and portends worse prognosis and higher mortality [2,3]. Additionally, such occlusion is often accompanied by hypotension and 
conduction atrioventricular disturbances [4]. Therefore, to decide optimal treatment and management of such patients, it is extremely important to recognize the location of the culprit vessel on the inferoposterior wall, as shown in Fig. 1.

The goal of this study was to find out the ECG criteria to predict proximal RCA occlusion as screening test before performing a reverse ECG or echocardiography.

\section{METHODS}

\section{Study population}

In our tertiary hospital, the ED has an annual volume of 32,000 patients and serves a primarily urban population. We retrospectively analyzed electrocardiographic and angiographic findings of consecutive patients admitted to the ED between March 2005 and December 2013 using a case control design. We reviewed 945 patients admitted to the ED during this period who diagnosed acute coronary syndrome (ACS) and underwent coronary angiography. Among them, 48 were diagnosed as having a pure pRCA lesion by direct percutaneous coronary intervention (PCI). These 48 patients were compared with a control group of 110 patients who were diagnosed as having either a pure mid to distal RCA lesion (57 patients) or a pure left circumflex artery (LCX) lesion (53 patients). We enrolled these 158 consecutive patients with an inferoposterior wall MI who fulfilled the following criteria: (1) absence of conditions precluding the evaluation of ST-segment changes on ECG (bundle branch block, ventricular rhythm, and artifacts); (2) fully assessable electrocardiography on admission; and (3) no mixed lesions involving two or more coronary arteries.

\section{Electrocardiographic analysis}

On admission, ECGs were recorded with standard 12 leads at a paper speed of $25 \mathrm{~mm} / \mathrm{sec}$ and an amplification of $10 \mathrm{~mm} / \mathrm{mV}$. All ECGs were reviewed independently by two physicians blinded to patient clinical and angio-
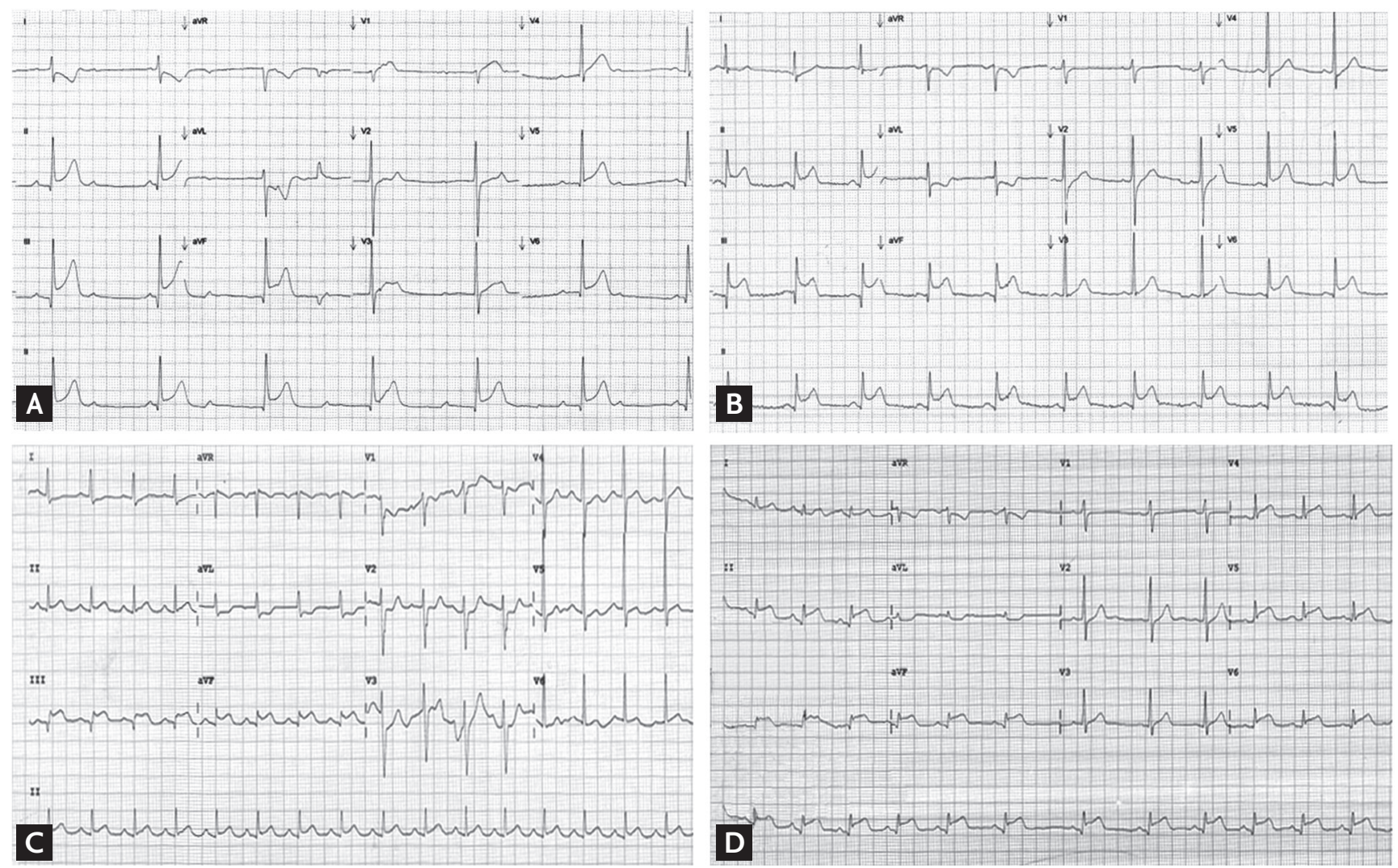

Figure 1. Electrocardiograms of each culprit lesion with single coronary artery stenosis. (A) Proximal right coronary artery (RCA) with 95\% stenosis. (B) Distal RCA with 100\% stenosis. (C) Proximal left circumflex artery (LCX) with 100\% stenosis. (D) Distal LCX with 100\% stenosis. 
graphic data. The isoelectric line was defined as the level of the preceding TP segment. ST-segment deviation was measured manually at $60 \mathrm{~ms}$ (ST60) after the end of the QRS complex (J point) by two investigators who were blinded to all clinical and angiographic findings. ECGs were magnified by a software (PicPick version 4 for Windows, Wiziple Inc., Korea) that displayed an enlarged portion of the ECG with more than 10 times magnification.

The variable, discrepancy of ST-segment deviation in leads III and II, was measured because it is known that a greater ST elevation in lead III than lead II predicted pRCA occlusion rather than mid to distal RCA or LCX occlusion [5]. The variable used absolute values from leads III and II to allow for minus values of ST-segment in each lead.

In addition, amplitudes and axes of ST vectors in leads were investigated. Based on registration of the heart's vector loops in three perpendicular directions (X, Y and $\mathrm{Z})$, a ST vector magnitude was calculated by simple geometry and direction of the ST vector that was directed toward the area of predominant myocardial injury was determined in two 2-dimensional planes as follows [6]: ST vector magnitude $=\sqrt{ }\left(X^{2}+Y^{2}+Z^{2}\right)$.

The frontal plane in limb leads was based on the axes of leads I and aVF. The horizontal plane in precordial leads was based on the axes of leads I and V2.

\section{Coronary angiography}

During the study period, PCI was performed when patients suspected of ACS had more than one of the following: ongoing chest pain; dynamic ST-T change; troponin elevation; unstable hemodynamics; an abnormal echocardiographic finding, such as regional wall motion abnormality or heart failure; prior PCI, coronary artery bypass graft; or a high risk score. Based on PCI, occlusion of the RCA or LCX supplying the inferoposterior wall was defined as a $70 \%$ or more narrowing of the luminal diameter of the coronary artery. The pRCA was defined as the coronary segment proximal to the first RV branch according to the American College of Cardiology/American Heart Association guidelines [7].

\section{Statistical analyses}

To determine interrater reliability for interpreting ECG, the arithmetic mean for each investigator was summa- rized by calculating the Spearman's correlation coefficient for each lead. To evaluate relationships between the ECG of cases, (pure pRCA lesions) and controls (LCX or mid or distal RCA lesions, except left anterior descending artery [LAD]), all categorical independent variables were analyzed using Fisher exact test; and the Mann-Whitney $U$ test was applied to all continuous independent variables. The significance of such relationships was tested through binary logistic regression analysis. Receiver operating characteristic (ROC) curves were employed to find optimal cut-off values and area under the curve (AUC) values for independent factors related to pRCA lesion according to various conditions. All calculations relied on standard software (SPSS version 21 for Windows, IBM Co., Armonk, NY, USA), with statistical significance set at $p<0.05$.

\section{RESULTS}

Based on PCI results, 158 patients were diagnosed with inferoposterior MI involving either the RCA or LCX. The mean age was $64 \pm 13$ years with male predominance (117 males, $74 \%)$. The mean time from angina onset to the ECG recording was $352 \pm 107.9$ minutes. Chest pain continued for $\geq 20$ minutes in 87 patients $(55 \%)$. The mean value of troponin I was $0.94 \pm 1.026$. Baseline characteristics of the case and control groups were compared in Table 1, but there were no significant difference between the pRCA group and controls.

ECG measurements by two independent physicians were significantly had reliability $(p<0.001)$. The intraclass correlation coefficients among ECG leads varied, range 0.85 to 0.97 .

Table 2 shows the details of the ECG characteristics of three patient groups. When the cases (pure pRCA lesion) were compared with the controls, ST changes measured at $60 \mathrm{~ms}$ after point $\mathrm{J}$ were prominent in leads I and $\mathrm{V}_{1}$; there were no differences in measurements at other leads, as shown in Table 3. These findings indicated that both ST-segment depression in lead I and ST-segment elevation in $\mathrm{V} 1$ are important regardless of whether ST-segment values in leads II, III and aVF are positive or negative. Mean ST deviation of the three patient groups categorized by location of the coronary lesions (pRCA lesion, mid to distal RCA lesion and LCX lesion) were 
Table 1. Baseline demographic and clinical characteristics of cases and controls

\begin{tabular}{lccc}
\hline Characteristic & Proximal RCA $(\mathrm{n}=48)$ & The other RCA and LCX $(\mathrm{n}=110)$ & $p$ value \\
\hline Male sex & $36(75)$ & $81(73.6)$ & 0.857 \\
Age, yr & $66(41-89)$ & $358 \pm 112.63$ & 0.082 \\
Time from symptom onset to first & $338.04 \pm 96.02$ & & 0.269 \\
ECG, min & & $60(54.5)$ & 0.843 \\
Angina $>$ 20 minutes & $27(56.3)$ & $28(25.5)$ & 0.310 \\
Old MI > 3 months & $16(33.3)$ & $48(43.6)$ & 0.087 \\
Diabetes mellitus & $14(29.2)$ & $67(60.9)$ & 0.301 \\
Hypertension & $25(52.1)$ & $57(71.3)$ & 0.652 \\
Hypercholesterolemia & $23(47.9)$ & $27(24.5)$ & 0.161 \\
Smoking & $17(35.4)$ & $69.3 \pm 15.94$ & 0.234 \\
HR on admission, beats/min & $68.3 \pm 23.02$ & $122.6 \pm 16.65$ & 0.131 \\
SBP on admission, mmHg & $119.3 \pm 19.95$ & $0.85 \pm 0.84$ & 0.462 \\
TnI on admission, ng/mL & $1.15 \pm 1.35$ & $168 \pm 146.79$ & 0.581 \\
CK-MB on admission, U/L & $145.9 \pm 86.35$ & & \\
\hline
\end{tabular}

Values are presented as number (\%), median (range), or mean $\pm \mathrm{SD}$.

RCA, right coronary artery; LCX, left circumflex artery; ECG, electrocardiogram; MI, myocardial infarction; HR, heart rate; SBP, systolic blood pressure; TnI, troponin I; CK-MB, creatine kinase-MB.

Table 2. Characteristics of electrocardiogram of three patient groups categorized by the location of the coronary artery lesion

\begin{tabular}{|c|c|c|c|c|}
\hline Characteristic & Proximal RCA $(n=48)$ & The mid RCA below $(\mathrm{n}=57)$ & $\operatorname{LCX}(\mathrm{n}=53)$ & $p$ value \\
\hline \multicolumn{5}{|l|}{ ST deviation in lead } \\
\hline $\mathrm{I}, \mathrm{mm}$ & $-0.68 \pm 0.83$ & $-0.22 \pm 0.7$ & $-0.28 \pm 0.53$ & 0.002 \\
\hline $\mathrm{II}, \mathrm{mm}$ & $0.4 \pm 1.12$ & $0.67 \pm 1.87$ & $-0.06 \pm 0.66$ & 0.016 \\
\hline III, mm & $1.18 \pm 1.65$ & $1.06 \pm 2.11$ & $0.29 \pm 0.81$ & 0.011 \\
\hline $\mathrm{aVR}, \mathrm{mm}$ & $0.18 \pm 0.57$ & $-0.04 \pm 1.04$ & $0.25 \pm 0.56$ & 0.133 \\
\hline $\mathrm{aVL}, \mathrm{mm}$ & $-0.74 \pm 1.05$ & $-0.59 \pm 1.26$ & $-0.2 \pm 0.6$ & 0.021 \\
\hline $\mathrm{aVF}, \mathrm{mm}$ & $0.81 \pm 1.38$ & $0.71 \pm 2.04$ & $0.08 \pm 0.71$ & 0.029 \\
\hline $\mathrm{V} 1, \mathrm{~mm}$ & $0.97 \pm 0.93$ & $0.09 \pm 1.39$ & $0.6 \pm 1.02$ & 0.001 \\
\hline $\mathrm{V} 2, \mathrm{~mm}$ & $0.94 \pm 1.85$ & $0.51 \pm 2.44$ & $0.9 \pm 1.68$ & 0.475 \\
\hline $\mathrm{V}_{3}, \mathrm{~mm}$ & $0.96 \pm 1.72$ & $0.72 \pm 1.75$ & $0.7 \pm 1.7$ & 0.696 \\
\hline $\mathrm{V}_{4}, \mathrm{~mm}$ & $0.39 \pm 1.26$ & $0.19 \pm 1.34$ & $-0.03 \pm 1.22$ & 0.252 \\
\hline $\mathrm{V}_{5}, \mathrm{~mm}$ & $-0.13 \pm 1.29$ & $0.15 \pm 1.27$ & $-0.24 \pm 1.1$ & 0.266 \\
\hline $\mathrm{V} 6, \mathrm{~mm}$ & $-0.25 \pm 1.39$ & $0.2 \pm 1.35$ & $-0.11 \pm 0.92$ & 0.165 \\
\hline $\mathrm{ST}$ in $\mathrm{III}>\mathrm{ST}$ in $\mathrm{II}^{\mathrm{a}}$ & $19(59 \cdot 4)$ & $35(61.4)$ & $13(68.4)$ & 0.029 \\
\hline \multicolumn{5}{|c|}{ Amplitude and axis of ST vectors in lead } \\
\hline Vector magnitude, mm & $2.4 \pm 1.5$ & $2.44 \pm 2.4$ & $1.7 \pm 2.3$ & 0.062 \\
\hline Frontal axis, $^{\circ}$ & $162.2 \pm 67.7$ & $174.5 \pm 75 \cdot 9$ & $170.7 \pm 80.6$ & 0.698 \\
\hline Horizontal axis, $^{\circ}$ & $147 \cdot 5 \pm 66.8$ & $141.1 \pm 73.1$ & $137 \pm 71.2$ & 0.753 \\
\hline
\end{tabular}

Values are presented as mean $\pm \mathrm{SD}$ or number (\%).

${ }^{\mathrm{a}}$ The absolute value of ST-segment deviation in leads II, III was applied. 
Table 3. Univariate analysis of the electrocardiography findings in the cases (pRCA lesion) compared with the controls (either the mid or distal RCA lesion or left circumflex artery lesions)

\begin{tabular}{|c|c|c|c|c|}
\hline \multirow{2}{*}{ Variable } & \multirow{2}{*}{ OR } & \multirow{2}{*}{$p$ value $(95 \% \mathrm{CI})$} & \multicolumn{2}{|c|}{$\mathrm{II}>\mathrm{o}, \mathrm{III}>\mathrm{O}, \mathrm{aVF}>\mathrm{O}^{\mathrm{a}}$} \\
\hline & & & OR & $p$ value $(95 \% \mathrm{CI})$ \\
\hline $\mathrm{I}, \mathrm{mm}$ & 0.419 & $0.001(0.245-0.717)$ & 0.316 & $0.003(0.148-0.673)$ \\
\hline $\mathrm{II}, \mathrm{mm}$ & 1.040 & $0.754(0.816-1.325)$ & 0.897 & $0.573(0.615-1.309)$ \\
\hline III, mm & 1.188 & $0.098(0.969-1.457)$ & 1.157 & $0.294(0.882-1.517)$ \\
\hline $\mathrm{aVR}, \mathrm{mm}$ & 1.139 & $0.575(0.723-1.794)$ & 2.031 & $0.097(0.879-4.688)$ \\
\hline $\mathrm{aVL}, \mathrm{mm}$ & 0.733 & $0.063(0.529-1.016)$ & 0.848 & $0.432(0.562-1.280)$ \\
\hline $\mathrm{aVF}, \mathrm{mm}$ & 1.182 & $0.142(0.946-1.477)$ & 1.094 & $0.529(0.827-1.446)$ \\
\hline $\mathrm{V} 1, \mathrm{~mm}$ & 1.853 & $0.002(1.258-2.731)$ & 2.114 & $0.005(1.258-3.552)$ \\
\hline $\mathrm{V} 2, \mathrm{~mm}$ & 1.063 & $0.498(0.890-1.220)$ & 1.047 & $0.644(0.861-1.274)$ \\
\hline $\mathrm{V}_{3}, \mathrm{~mm}$ & 1.090 & $0.394(0.894-1.328)$ & 1.027 & $0.827(0.810-1.301)$ \\
\hline $\mathrm{V}_{4}, \mathrm{~mm}$ & 1.209 & $0.169(0.922-1.585)$ & 1.083 & $0.668(0.751-1.563)$ \\
\hline $\mathrm{V}_{5}, \mathrm{~mm}$ & 0.940 & $0.665(0.711-1.243)$ & 0.760 & $0.164(0.517-1.118)$ \\
\hline $\mathrm{V} 6, \mathrm{~mm}$ & 0.812 & $0.165(0.605-1.09)$ & 0.713 & $0.125(0.463-1.098)$ \\
\hline $\mathrm{ST}$ in $\mathrm{III}>\mathrm{ST}$ in $\mathrm{II}^{\mathrm{b}}$ & 2.593 & $0.013(1.221-5.507)$ & 9.750 & $0.033(1.201-79.165)$ \\
\hline Vector magnitude, mm & 1.092 & $0.338(0.913-1.306)$ & 1.023 & $0.818(0.844-1.240)$ \\
\hline Frontal axis, $^{\circ}$ & 0.998 & $0.418(0.994-1.003)$ & 1.013 & $0.074(0.999-1.028)$ \\
\hline Horizontal axis, $^{\circ}$ & 1.002 & 0.488 (0.997-1.007) & 1.003 & $0.342(0.997-1.010)$ \\
\hline
\end{tabular}

pRCA, proximal right coronary artery; OR, odds ratio; CI, confidence interval.

${ }^{a}$ If leads II, III and aVF were not > o mm, their values were not included in the analysis.

${ }^{\mathrm{b}}$ The absolute value of ST-segment deviation in leads II, III was applied.

Table 4. Analysis of variables categorized by cut-off values (ST-segment deviation in lead I $\leq-1.1$ mm defined by Youden's J index; in $\mathrm{V}_{1}>0.65 \mathrm{~mm}$ respectively) by multivariable logistic regression

\begin{tabular}{lcccc}
\hline Variable & OR & pvalue $(95 \%$ CI $)$ & \multicolumn{2}{c}{ II $>0$, III $>0, a V F>0^{a}$} \\
\cline { 3 - 5 } I & 3.55 & $0.01(1.348-9.346)$ & 3.516 & $0.052(0.987-12.526)$ \\
V1 & 3.2 & $0.002(1.508-6.796)$ & 6.589 & $0.001(2.088-20.798)$ \\
ST in III > ST in II & 4.64 & $0.031(1.084-5.524)$ & 7.892 & $0.075(0.813-70.895)$ \\
\hline
\end{tabular}

OR, odds ratio; CI, confidence interval.

${ }^{a}$ If leads II, III and aVF were not > o mm, their values were not included in the analysis.

${ }^{\mathrm{b}}$ The absolute value of ST-segment deviation in leads II, III was applied.

$-0.68,-0.22$, and $-0.28 \mathrm{~mm}$ in lead I and $0.97,0.09$, and $0.6 \mathrm{~mm}$ in lead $\mathrm{V} 1$, respectively, as shown in Table 2.

Greater ST-segment elevation in lead III than lead II was associated with the case group (pRCA lesion), but other variables that measured amplitude and axis vectors (ST vector magnitude, frontal axis, and horizontal axis), did not differ significantly between the cases and controls. ST deviation in leads I, V1 and greater ST-segment elevation in lead III than lead II differed significantly between cases and controls in multivariable anal- ysis; the odds ratios were 3.6, 3.2, and 4.6, respectively as seen in Table 4. However, when only patients with the positive ST-segment values in leads II, III, and aVF were included in the analysis, a greater ST-elevation in lead III than lead II was not significantly associated with pRCA lesion as shown that the $95 \%$ confidence interval of the odds ratio was wide, 0.813 to 70.895 . Perhaps this would be due to the small population included in this study. Nevertheless, the odds ratio was 7.9, displayed in Table 4. 

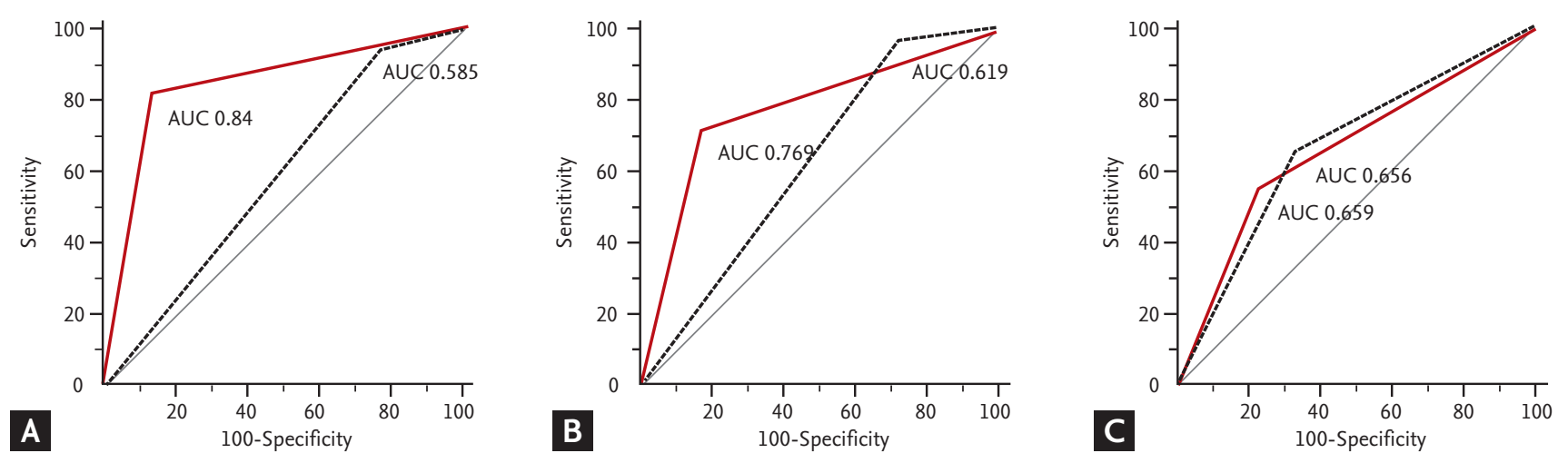

Figure 2. Comparison of receiver operating characteristic (ROC) curves between the values of lead I plus lead V1 and the values that ST-segment of lead III was more than lead II according to three conditions (the combined variable considered each ST deviation in lead I and V1 was ST depression in lead I > o mm and ST elevation in lead V1 > 0.5 mm by ROC curve). (A) The values of lead I < O mm + V1 > 0.5 mm were defined if leads II, III, and aVF were all > o mm (area under the curve [AUC; I + V1] 0.840 ; AUC [III > II], 0.585). (B) The values of lead I < O mm + V1 > 0.5 mm were defined regardless of whether leads II, III, and aVF were all $>0 \mathrm{~mm}$, and the ST-segment in lead III was compared to the ST-segment in lead II using |absolute value| (AUC [I + V1], 0.769 ; AUC [III > II], 0.619). (C) The values of lead I < o mm + V1 > 0.5 mm were defined if two contiguous leads of II, III, and aVF were < $1 \mathrm{~mm}$ and the ST-segment in lead III was compared to the ST-segment in lead II (absolute value) (AUC [I + V1], o.659; AUC [III + II], 0.656).

Fig. 2 shows that the variable combined each ST deviation of lead I and V1 was the most related with pRCA lesion. The most eligible value considered each ST deviation in lead I and V1 that was ST depression in lead I > 0 $\mathrm{mm}$ and ST elevation in lead $\mathrm{V}_{1}>0.5 \mathrm{~mm}$ by ROC curve was attained after more trial with combination of several cut-off values. The variable of lead I plus the variable of lead V1 had better diagnostic value than gap between ST deviation in lead III and II (AUC, o.84 vs. 0.585 ) if all ST-segment values of leads II, III, and aVF were positive. However, when patients with lower ST-segment values than $1 \mathrm{~mm}$ in leads II, III, and aVF were included the diagnostic value of this variable was decreased (AUC, 0.769 from 0.84 ). Especially, when having negative value of ST-segment in one of leads II, III, and aVF at least two variables combined had a weak association and lower AUC value related with pRCA stenosis than the variable of gap between ST deviation in lead III and II (AUC, 0.659 vs. 0.656 ).

\section{DISCUSSION}

In cases of inferoposterior MI due to occlusion of the pRCA, ST-segment changes in inferior leads (ST eleva- tion in leads II, III, and aVF) are the key to recognizing the injured area because the injury vector of the affected area is directed predominantly downward. However, because the injury vector is also directed slightly to the right it provokes ST depression in leads I and aVL. Also, RV injury caused by pRCA occlusion results in more inferior than posterolateral injury, producing a vector of injury that attenuates reciprocal ST depression (and may even lead to ST elevation) in leads V1 and V2, as well as making ST elevation more prominent in lead III than in lead II [8]. Hence, such a vectorial approach to interpretation of ST-segment changes may be useful for the discriminating pRCA lesions from other coronary lesions because with pRCA occlusion the ST injury vector is directed towards the right and anteriorly inferiorly [6].

Two case reports found that increased vector amplitude indicated a high probability of acute MI and that the amplitude and direction of ST injury vector were in concordance with the infarction area presented in SPECT (single positron emission computed tomography) $[6,9]$. However, in our study, the amplitude and direction of the ST injury vector were not able to discriminate between proximal and distal RCA lesion. Perhaps the vectors in ECG depend other characteristics, such as the progression of collateral vessels, degree of stenosis 
or extent of lesion in the endocardium or epicardium.

RV involvement, which may be diagnosed by detecting unexpected oxygen desaturation, hypotension, $\mathrm{V}_{4} \mathrm{R}$ in a reverse ECG and echocardiography, usually occurs because the RCA is occluded proximal to the RV branch, with associated acute inferior wall infarction. Less commonly, RV infarction results from occlusion of a dominant LCX in the setting of acute lateral wall MI; less than $10 \%$, of RV infarctions arise from LAD occlusion in acute anterior wall MI [10]. Rarely, isolated RV infarction originates from non-dominant RCA occlusion or marginal branch occlusion of the RCA $[10,11]$. In patients with RCA dominant circulation or co-dominant circulation, which occurs in about 90\%, their RCA perfuses the right ventricle, inferior-wall left ventricle and the lower part of the intraventricular septum. Therefore, occlusion of the pRCA will result in RV infarction and negatively affects prognosis.

The RV involvement that usually accompanies pRCA occlusion may be indicated by ST-segment changes in right precordial leads [12]. Isoelectric or ST-segment elevation in lead V1 was observed in 70\% of patients with pRCA occlusion and only in $13 \%$ of patients with distal occlusion. This criterion had 70\% sensitivity and $87 \%$ specificity for the detection of pRCA occlusion [13]. In another study similar to our data, both the amplitude of ST elevation in lead V1 (0.51 mm vs. $-0.56 \mathrm{~mm}, p<0.01$ ) and ST depression of lead I $(-1.57 \mathrm{~mm}$ vs. $-0.83 \mathrm{~mm}, p<$ o.o1) were significantly higher in proximal than in distal RCA occlusions and these criteria were used as screening test to distinguish proximal and distal RCA lesions, adding to the ST deviation in lead II, III and V3. They were incorporated into a score that distinguished proximal from distal stenosis of the RCA [14]. And ECGs indicating pRCA occlusion showed ST depression in lead I but isoelectric ST in lead $\mathrm{V}_{1}$ in a recent study [15].

On the other hand, one study reported ST elevation in lead V1 in ECG of patients with pRCA occlusion was more meaningful than with distal RCA occlusion (o mm vs. $-1.5 \mathrm{~mm}, p<0.01$ ) but obvious difference was not observed in lead I ( $-1 \mathrm{~mm}$ vs. $-1 \mathrm{~mm}, p=$ non-specific) [13]. Furthermore, a research report concluded that ST deviations in lead V1 were not related to pRCA occlusion [16].

The ratio of ST-segment elevation in lead II to ST elevation in lead III was the main ECG feature that discriminates LCX from RCA according to many authors, including Tierala et al. [17]. In addition, the comparison of ST elevation in leads II and III has been used a screening tool for right ventricular infarction (RVI). However, greater ST elevation in lead III than lead II is found to be sensitive (97\%) but relatively nonspecific compared to ST-segment elevation in lead V4R (56\% vs. $78 \%$, respectively) $[18,19]$. Furthermore, left dominance of coronary artery charged in $15 \%$ was an important contributing factor to the misclassification of culprit coronary artery and made a difference of ST elevation in leads II and III $[15,20]$. Meanwhile, the difference of ST elevation in lead II and III was showed as having meaning slightly in our analysis; however, ST elevation in lead V1 and ST depression in lead I were more discriminant features than the difference of ST elevation in lead II and III as a good predictor of lesions of the pRCA.

ST depression $>1 \mathrm{~mm}$ in lead aVL was very sensitive and specific for RV involvement in patients with acute MI compared to diagnosis of RVI based on changes in right precordial leads and ST elevation $>1 \mathrm{~mm}$ in lead $\mathrm{V}_{4} \mathrm{R}$ [21]. However, a correlation between ST depression in aVL and pRCA occlusion was not observed in our data.

The criterion of ST changes in I and V1 have had the highest accuracy for distinguishing pRCA versus dRCA or LCX occlusion although physician had thought that gap of ST deviation in III and II allowed prediction of the location of occlusion in pRCA. Therefore, this criterion of ST changes in I and V1 may be recommended to be applied in the emergency room as it may help to make a decision regarding the need of urgent management to RVI or advanced diagnostic tool like reverse ECG or echocardiography.

The major limitation of this study was the small number of patients. We only included highly selected patients with lesions in a single coronary artery and only included patients with lesions in the RCA and LCX, not with lesions located in the LAD. In our study, the lead $\mathrm{V}_{4} \mathrm{R}$ was not evaluated because reverse ECG data was frequently missing from patient records. In addition, the anatomical variation of individuals was not considered. The posterior descending and posterolateral branches depending on the dominant or non-dominant coronary vessel that perfuse the inferolateral sections of the left ventricle can originate from either the RCA or the LCX. The dominant branch might be able to have an effect 
on detecting pRCA occlusion or RVI in ECG. Although progression of collateral circulation due to chronic ischemia could affect features of the ECG, collateral circulation was not investigated.

In conclusion, the combination of ST elevation in lead V1 and ST depression in lead I respectively allow more accurate prediction of occlusion located in the pRCA than other criteria, such as the difference between ST elevation of leads II and III or vector direction and amplitude. In addition, ST deviation in leads V1 and I is related to RVI. Therefore, these variables could help to screen for RVI before performing reverse ECG and predicting prognosis.

\section{KEY MESSAGE}

1. Prediction of proximal right coronary artery (RCA) occlusion in 12 leads electrocardiogram (ECG) as a screening tool to predict it briefly before performing reverse ECG has clinical importance.

2. There was little evidence that amplitudes and axes of ST vectors in ECGs was indicating coronary lesion.

3. A combination of prominent ST depression in lead I and ST elevation in V1 will be suggested as better screening test than the difference between ST elevation of leads II and III for detecting the proximal RCA occlusion.

\section{Conflict of interest}

No potential conflict of interest relevant to this article was reported.

\section{Acknowledgments}

This work was supported by the Dong-A University Research Fund.

\section{REFERENCES}

1. Wright RS, Anderson JL, Adams CD, et al. 2011 ACCF/ AHA focused update of the guidelines for the management of patients with unstable angina/non-ST-elevation myocardial infarction (updating the 2007 guideline): a report of the American College of Cardiology Founda-
tion/American Heart Association Task Force on Practice Guidelines. Circulation 2011;123:2022-2060.

2. Berger PB, Ryan TJ. Inferior myocardial infarction: highrisk subgroups. Circulation 1990;81:401-411.

3. Zehender M, Kasper W, Kauder E, et al. Right ventricular infarction as an independent predictor of prognosis after acute inferior myocardial infarction. N Engl J Med 1993;328:981-988.

4. Hanzel GS, Merhi WM, O'Neill WW, Goldstein JA. Impact of mechanical reperfusion on clinical outcome in elderly patients with right ventricular infarction. Coron Artery Dis 2006;17:517-521.

5. Zimetbaum PJ, Krishnan S, Gold A, Carrozza JP 2nd, Josephson ME. Usefulness of ST-segment elevation in lead III exceeding that of lead II for identifying the location of the totally occluded coronary artery in inferior wall myocardial infarction. Am J Cardiol 1998;81:918-919.

6. Andersen MP, Terkelsen CJ, Struijk JJ. The ST compass: spatial visualization of ST-segment deviations and estimation of the ST injury vector. J Electrocardiol 2009;42:181-189.

7. Scanlon PJ, Faxon DP, Audet AM, et al. ACC/AHA guidelines for coronary angiography: a report of the American College of Cardiology/American Heart Association Task Force on practice guidelines (Committee on Coronary Angiography). Developed in collaboration with the Society for Cardiac Angiography and Interventions. J Am Coll Cardiol 1999;33:1756-1824.

8. Fiol M, Cygankiewicz I, Carrillo A, et al. Value of electrocardiographic algorithm based on "ups and downs" of ST in assessment of a culprit artery in evolving inferior wall acute myocardial infarction. Am J Cardiol 2004;94:709714 .

9. Fesmire FM, Eriksson SV, Stout PK, Wojcik JF, Wharton DR. Use of baseline ST-vector magnitude to identify electrocardiographic injury in patients with suspected acute myocardial infarction. Am J Emerg Med 2002;20:535-540.

10. Rotondo N, Pollack ML, Chan TC, Brady WJ, Harrigan RA. Electrocardiographic manifestations: acute inferior wall myocardial infarction. J Emerg Med 2004;26:433-440.

11. Fijewski TR, Pollack ML, Chan TC, Brady WJ. Electrocardiographic manifestations: right ventricular infarction. J Emerg Med 2002;22:189-194.

12. Wellens $H J$. The value of the right precordial leads of the electrocardiogram. N Engl J Med 1999;340:381-383.

13. Fiol M, Carrillo A, Cygankiewicz I, et al. New criteria 
based on ST changes in 12-lead surface ECG to detect proximal versus distal right coronary artery occlusion in a case of acute inferoposterior myocardial infarction. Ann Noninvasive Electrocardiol 2004;9:383-388.

14. Jim MH, Tsui KL, Yiu KH, et al. Jeopardised Inferior Myocardium (JIM) score: an arithmetic electrocardiographic score to predict the infarct-related artery in inferior myocardial infarction. Ann Acad Med Singapore 2012;41:300-304.

15. Gregg RE, Fiol-Sala M, Nikus KC, et al. Automated discrimination of proximal right coronary artery occlusion from middle-to-distal right coronary artery occlusion and left circumflex occlusion in ST-elevation myocardial infarction. J Electrocardiol 2012;45:343-349.

16. Wong CK, Freedman SB. Precordial ST change and site of the infarct-related lesion in right coronary artery-related inferior wall acute myocardial infarction. Am J Cardiol 1995;75:942-943.

17. Tierala I, Nikus KC, Sclarovsky S, Syvanne M, Eskola M; HAAMU Study Group. Predicting the culprit artery in acute ST-elevation myocardial infarction and introducing a new algorithm to predict infarct-related artery in infe- rior ST-elevation myocardial infarction: correlation with coronary anatomy in the HAAMU Trial. J Electrocardiol 2009;42:120-127.

18. Saw J, Davies C, Fung A, Spinelli JJ, Jue J. Value of ST elevation in lead III greater than lead II in inferior wall acute myocardial infarction for predicting in-hospital mortality and diagnosing right ventricular infarction. Am J Cardiol 2001;87:448-450.

19. Andersen HR, Nielsen D, Falk E. Right ventricular infarction: diagnostic value of ST elevation in lead III exceeding that of lead II during inferior/posterior infarction and comparison with right-chest leads $\mathrm{V}_{3} \mathrm{R}$ to $\mathrm{V}_{7} \mathrm{R}$. Am Heart J 1989;117:82-86.

20. Tahvanainen M, Nikus KC, Holmvang L, et al. Factors associated with failure to identify the culprit artery by the electrocardiogram in inferior ST-elevation myocardial infarction. J Electrocardiol 2011;44:495-501.

21. Turhan H, Yilmaz MB, Yetkin E, et al. Diagnostic value of aVL derivation for right ventricular involvement in patients with acute inferior myocardial infarction. Ann Noninvasive Electrocardiol 2003;8:185-188. 